\title{
Edoxaban noninferior to warfarin in patients with AF
}

Two different dose regimens of the direct oral factor Xa inhibitor edoxaban have been shown to be noninferior to warfarin for stroke prevention in patients with atrial fibrillation (AF). These findings from the ENGAGE AF-TIMI 48 trial were presented at the 2013 AHA Scientific Sessions and simultaneously published in the New England Journal of Medicine.

\section{4 ...the conduct of the ENGAGE AF-TIMI 48 trial was exemplary 77}

"Edoxaban is a more-targeted, simpler approach to preventing blood clots [than warfarin]," said lead investigator Robert Giugliano in a news conference at the AHA meeting. "It inhibits the body's ability to form a clot at a very critical juncture of the clotting pathway and behaves in a more-predictable way." Edoxaban is now the fourth novel oral anticoagulant to be compared with warfarin in patients with AF.
This multinational, randomized, doubleblind, double-dummy trial involved 21,105 patients recruited from 1,393 centres in 46 countries. Trial participants were aged $\geq 21$ years, had AF documented within the 12 months before study enrolment, and a $\mathrm{CHADS}_{2}$ score $\geq 2$. Patients were randomly assigned to receive warfarin (dosed to achieve an international normalized ratio of 2-3), high-dose edoxaban (60 mg), or low-dose edoxaban ( $30 \mathrm{mg}$ ), all given once daily. A 50\% reduction in the dose of edoxaban was permitted for patients with renal dysfunction, body weight $<60 \mathrm{~kg}$, or concomitant use of a P-glycoprotein inhibitor. Around one-third of the patients in each group discontinued the study drug during the trial. Consent for follow-up was withdrawn by 244 patients; however, only one patient was actually lost to follow-up. The median time in the therapeutic range for patients receiving warfarin was $68.4 \%$.

The annul rate of the primary end point (stroke or systemic embolism) was $1.50 \%, 1.18 \%$, and $1.61 \%$ in the warfarin, high-dose, and low-dose edoxaban groups, respectively. Both dose regimens of edoxaban were noninferior to warfarin (high dose: $P<0.001$; low dose: $P=0.005$ ). In a prespecified analysis of the intentionto-treat population, neither regimen of edoxaban was shown to be superior to warfarin. The rates of haemorrhagic stroke and cardiovascular death were significantly lower for both regimens of edoxaban than for warfarin. The annual rate of major bleeding (the primary safety end point) was significantly lower with both high-dose $(2.75 \%)$ and low-dose $(1.61 \%)$ edoxaban than with warfarin (3.43\%; HR 0.80 for comparison with high-dose edoxaban, $P<0.001$; HR 0.47 for comparison with low-dose edoxaban, $P<0.001)$. Commenting at the AHA Scientific Sessions, Elaine Hylek from Boston University School of Medicine, USA, said that "the conduct of the ENGAGE AF-TIMI 48 trial was exemplary".

Alexandra Roberts

Original article Giugliano, R. P. et al. Edoxaban versus warfarin in patients with atrial fibrillation. N. Engl. J. Med. doi:10.1056/NEJMoa1310907 